\title{
Deformation stability of BRST-quantization
}

\author{
M. Dütsch*and K. Fredenhagen \\ Institut für Theoretische Physik \\ Universität Hamburg \\ 149, Luruper Chaussee \\ D-22761 Hamburg, Germany \\ duetsch@mail.desy.de, fredenha@@x4u2.desy.de
}

\begin{abstract}
To avoid the problems which are connected with the long distance behavior of perturbative gauge theories we present a local construction of the observables which does not involve the adiabatic limit. First we construct the interacting fields as formal power series by means of causal perturbation theory. The observables are defined by BRST invariance where the BRST-transformation $\tilde{s}$ acts as a graded derivation on the algebra of interacting fields. Positivity, i.e. the existence of Hilbert space representations of the local algebras of observables is shown with the help of a local Kugo-Ojima operator $Q_{\text {int }}$ which implements $\tilde{s}$ on a local algebra and differs from the corresponding operator $Q$ of the free theory. We prove that the Hilbert space structure present in the free case is stable under perturbations. All assumptions are shown to be satisfied in QED in a finite spatial volume with suitable boundary conditions. As a by-product we find that the BRST-quantization is not compatible with periodic boundary conditions for massless free gauge fields.
\end{abstract}

PACS. 11.15.-q Gauge field theories, 11.15.Bt General properties of perturbation theory

\section{Introduction}

The long distance behavior of nonabelian perturbative gauge theories is plagued by serious problems. In massless theories there appear infrared divergences in the adiabatic limit $g \rightarrow$ const. of the S-matrix, where $g$ is a space-time dependent coupling 'constant'. In QED these divergences are logarithmic and cancel in the cross section. (This is proven at least at low orders of the perturbation series [15].) Moreover, Blanchard and Seneor [2 proved that the adiabatic limit of Green's and Wightman functions exists for QED. But in nonabelian gauge

\footnotetext{
*Work supported by the Alexander von Humboldt Foundation
} 
theories the divergences are worse. Perturbation theory seems to be not able to describe the long distance properties of these models ("confinement"). In massive theories the infrared divergences are absent, but e.g. in the electroweak theory an S-matrix formalism suffers from the instability of some particles, e.g. the W-, Z-bosons and the muons and taus. States containing such particles belong to the physical state space, but they cannot appear as asymptotic states of the S-matrix for $t \rightarrow \pm \infty$.

Our way out is to construct the observables locally. We consider a fixed, open double cone $\mathcal{O} \subset \mathbf{R}^{4}$. The coupling 'constant' $g$ has compact support and takes a constant value on $\mathcal{O}$

$$
g \in \mathcal{D}\left(\mathbf{R}^{4}\right), \quad g(x)=e=\text { const. }, \quad \forall x \in \mathcal{O} .
$$

The interacting fields are defined by Bogoliubov's formula [4]

$$
\left.A_{\text {int } \mathcal{L}}(x) \stackrel{\text { def }}{=} \frac{\delta}{i \delta h(x)} S(\mathcal{L})^{-1} S(\mathcal{L}+h A)\right|_{h=0},
$$

and the time ordered products $T\left(\mathcal{L}\left(x_{1}\right) \ldots \mathcal{L}\left(x_{n}\right)\right)$, which appear in the S-matrix

$$
S(\mathcal{L})=\sum_{n=0}^{\infty} \frac{i^{n}}{n !} \int d^{4} x_{1} \ldots d^{4} x_{n} T\left(\mathcal{L}\left(x_{1}\right) \ldots \mathcal{L}\left(x_{n}\right)\right)
$$

are constructed by means of causal perturbation theory 4 , 6, 12, 15, 17. The interacting fields $A_{\text {int } \mathcal{L}}(x)$ ( $A$ is a Wick polynomial of incoming fields) are formal power series of operator valued distributions on a dense invariant domain $\mathcal{D}$ in the Fock space of incoming fields. They depend on an interaction Lagrangian $\mathcal{L}$ which is a Wick polynomial of incoming fields with testfunctions $g \in \mathcal{D}\left(\mathbf{R}^{4}\right)$ as coefficients.

The crucial observation is that the dependence of the interacting fields on the interaction Lagrangian is local, in the sense that Lagrangians $\mathcal{L}_{1}$ and $\mathcal{L}_{2}$ which differ only within a closed region which does not intersect the closure of $\mathcal{O}$, lead to unitarily equivalent fields within $\mathcal{O}$, i.e. there exists a unitary formal power series $V$ of operators on $\mathcal{D}$ such that

$$
V A_{\text {int } \mathcal{L}_{1}}(x) V^{-1}=A_{\text {int } \mathcal{L}_{2}}(x), \quad \forall x \in \mathcal{O}
$$

and $V$ does not depend on $A[6]$. The proof of (1.4) relies on the causal factorization of the time ordered products.

The field algebra $\tilde{\mathcal{F}}(\mathcal{O})$ which is generated by

$$
\left\{A_{\text {int } \mathcal{L}}(f)=\int d^{4} x A_{\text {int } \mathcal{L}}(x) f(x) \mid f \in \mathcal{D}(\mathcal{O})\right\}
$$

is up to unitary equivalence uniquely determined by $\left.g\right|_{\mathcal{O}}$. Since $\mathcal{O}$ is arbitrary, the full net of local algebras can be constructed without ever performing the adiabatic limit $g \rightarrow$ constant. 
In gauge theories the (local) algebras of interacting fields contain unphysical fields like vector potentials and ghosts. They can be eliminated by the BRST formalism. But it remains to show that the algebra of observables can be (nontrivially) represented on a Hilbert space.

In the free theory positivity can be verified by an explicit calculation. Formally, in the adiabatic limit, the positivity is hence valid also for the interacting theory. We show, that for a localized interaction (i.e. before the adiabatic limit), the physical Hilbert space can be obtained as a deformation of the free one (sect. 2). The construction relies on some assumptions, which are verified for the example of QED (sect. 3). To avoid a volume divergence in $Q_{\text {int }}$ we embed our double cone $\mathcal{O}$ into the cylinder $\mathbf{R} \times C_{L}$, where $\mathbf{R}$ denotes the time axis and $C_{L}$ is a cube of length $L$. In sect. 4 we point out the importance of a suitable choice of boundary conditions for the BRST-quantization of massless free gauge fields on $\mathbf{R} \times C_{L}$.

We hope that due to its local character, our construction can be generalized to curved space-times, continuing the program of [6, 7].

\section{Connection of observable algebras and field algebras in perturbative gauge theories}

\subsection{Local construction of observables in gauge theories and representation in the physical pre Hilbert space}

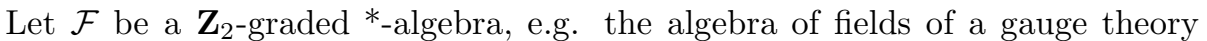
where the $\mathbf{Z}_{2}$-gradiation is $(-1)^{\delta(F)}$ with the ghost number $\delta(F)$. To get rid of the unphysical fields, we use the BRST-transformation $s$ [1], which is a graded derivation on $\mathcal{F}$ with $s^{2}=0$ and $s\left(F^{*}\right)=-(-1)^{\delta(F)} s(F)^{*}$.

The kernel of $s, \mathcal{A}_{0}:=s^{-1}(0)$, is a $*$-subalgebra of $\mathcal{F}$ and $\mathcal{A}_{00}:=s(\mathcal{F})$ is a 2 -sided ideal in $\mathcal{A}_{0}$. Hence we may define the algebra of observables as the quotient

$$
\mathcal{A} \stackrel{\text { def }}{=} \frac{\mathcal{A}_{0}}{\mathcal{A}_{00}} .
$$

We ask now under which conditions $\mathcal{A}$ has a nontrivial *-representation by operators on a pre Hilbert space. For this purpose we work with the KugoOjima formalism [14]. We assume that $\mathcal{F}$ has a faithful representation on an inner product space $(\mathcal{K},<., .>)$ such that $\left\langle F^{*} \phi, \psi\right\rangle=\langle\phi, F \psi\rangle, \forall F \in \mathcal{F}$, and that $s$ is implemented by an operator $Q$ on $\mathcal{K}$, i.e.

$$
s(F)=Q F-(-1)^{\delta(F)} F Q,
$$

such that

$$
<Q \phi, \psi>=<\phi, Q \psi>\quad \text { and } \quad Q^{2}=0 .
$$

Note that if the inner product on $\mathcal{K}$ is positive definite, we find $\langle Q \phi, Q \phi\rangle=$

$<\phi, Q^{2} \phi>=0$, hence $Q=0$ and thus also $s=0$. Hence for nontrivial $s$ the inner product must necessarily be indefinite. 
Let $\mathcal{K}_{0} \stackrel{\text { def }}{=} \operatorname{Ke} Q$ be the kernel and $\mathcal{K}_{00}$ the range of $Q$. Because of $Q^{2}=0$ we have $\mathcal{K}_{00} \subset \mathcal{K}_{0}$. We assume:

$$
\text { (Positivity) (i) } \quad<\phi, \phi>\geq 0 \quad \forall \phi \in \mathcal{K}_{0},
$$

and (ii) $\phi \in \mathcal{K}_{0} \wedge\left\langle\phi, \phi>=0 \Longrightarrow \phi \in \mathcal{K}_{00}\right.$.

Then

$$
\mathcal{H} \stackrel{\text { def }}{=} \frac{\mathcal{K}_{0}}{\mathcal{K}_{00}}, \quad<\left[\phi_{1}\right],\left[\phi_{2}\right]>_{\mathcal{H}}: \stackrel{\text { def }}{=}<\psi_{1}, \psi_{2}>_{\mathcal{K}}, \quad \psi_{j} \in\left[\phi_{j}\right]:=\phi_{j}+\mathcal{K}_{00}
$$

is a pre Hilbert space and

$$
\pi([A])[\phi] \stackrel{\text { def }}{=}[A \phi]
$$

is a representation on $\mathcal{H}\left(\right.$ where $\left.A \in \mathcal{A}_{0}, \phi \in \mathcal{K}_{0},[A]:=A+\mathcal{A}_{00}\right)$ [8].

\subsection{Stability under deformations}

It is gratifying that the described structure is stable under deformations, e.g. by turning on the interaction. Let $\mathcal{K}$ be fixed and replace $F \in \mathcal{F}$ by a formal power series $\tilde{F}=\sum_{n} g^{n} F_{n}$ with $F_{0}=F$ and $F_{n} \in \mathcal{F}, \delta\left(F_{n}\right)=$ const. In the same way replace $s$ and $Q$ by formal power series $\tilde{s}=\sum_{n} g^{n} s_{n}, \tilde{Q}=\sum_{n} g^{n} Q_{n}$ with $s_{0}=s, Q_{0}=Q$ and

$$
\tilde{s}^{2}=0, \quad \tilde{Q}^{2}=0, \quad<\tilde{Q} \phi, \psi>=<\phi, \tilde{Q} \psi>\quad \text { and } \quad \tilde{s}(\tilde{F})=\tilde{Q} \tilde{F}-(-1)^{\delta(\tilde{F})} \tilde{F} \tilde{Q}
$$

We can then define $\tilde{\mathcal{A}} \stackrel{\text { def }}{=} \frac{\mathrm{Ke} \tilde{s}}{\mathrm{Ra}} . \mathcal{K}_{0}$ and $\mathcal{K}_{00}$ have to be replaced by formal power series $\tilde{\mathcal{K}}_{0}:=\operatorname{Ke} \tilde{Q}$ and $\tilde{\mathcal{K}}_{00}:=\operatorname{Ra} \tilde{Q}$ with coefficients in $\mathcal{K}$. Due to the above result $(2.6)$, the algebra $\tilde{\mathcal{A}}$ has a natural representation on $\tilde{\mathcal{H}} \stackrel{\text { def }}{=} \frac{\tilde{\mathcal{K}}_{0}}{\tilde{\mathcal{K}}_{00}}$. The inner product on $\mathcal{K}$ induces an inner product on $\tilde{\mathcal{H}}$ which assumes values in the formal power series over $\mathbf{C}$. We adopt the point of view that a formal power series $\tilde{b}=\sum_{n} g^{n} b_{n}, b_{n} \in \mathbf{C}$ is positive if there is another formal power series $\tilde{c}=\sum_{n} g^{n} c_{n}, c_{n} \in \mathbf{C}$ with $\tilde{c}^{*} \tilde{c}=\tilde{b}$, i.e. $b_{n}=\sum_{k=0}^{n} \bar{c}_{k} c_{n-k}$. (cf. [5])

The assumptions concerning the positivity of the inner product are automatically fulfilled for the deformed theory, if they hold true in the undeformed model $[8]$.

Theorem 1: Let the positivity assumption (2.4) be fulfilled in zeroth order. Then

(i) $<\tilde{\phi}, \tilde{\phi}>\geq 0 \quad \forall \tilde{\phi} \in \tilde{\mathcal{K}}_{0}$,

(ii) $\tilde{\phi} \in \tilde{\mathcal{K}}_{0} \wedge \quad\left\langle\tilde{\phi}, \tilde{\phi}>=0 \quad \Longrightarrow \quad \tilde{\phi} \in \tilde{\mathcal{K}}_{00}\right.$.

(iii) For every $\phi \in \mathcal{K}_{0}$ there exists a power series $\tilde{\phi} \in \tilde{\mathcal{K}}_{0}$ with $(\tilde{\phi})_{0}=\phi$.

(iv) Let $\pi$ and $\tilde{\pi}$ be the representations (2.6) of $\mathcal{A}, \tilde{\mathcal{A}}$ on $\mathcal{H}, \tilde{\mathcal{H}}$ respectively. Then $\tilde{\pi}(\tilde{A}) \neq 0$ if $\pi\left(A_{0}\right) \neq 0$.

From parts (i) and (ii) we conclude that $\tilde{\mathcal{H}}=\frac{\tilde{\mathcal{K}}_{0}}{\mathcal{K}_{00}}$ is a perturbative analog of a (pre) Hilbert where the scalar product assumes values in the formal power 
series over $\mathbf{C}$. Note that $\phi \rightarrow \tilde{\phi}$ is non-unique and this holds also true for the induced relation between $\mathcal{H}$ and $\tilde{\mathcal{H}}$. A consequence of part (i) of the theorem is the positivity of the Wightman distributions of $\tilde{s}$-invariant fields [8].

\section{Verification of the assumptions in models}

Kugo-Ojima 14] argue that at asymptotically early times the interacting fields tend to the free incoming fields. Since $Q_{\text {int }} \stackrel{\text { def }}{=} \tilde{Q}$ is conserved, it coincides with the free one $Q=Q_{0}$. Hence it is sufficient to check the assumptions for the free theory. But the BRST-current (i.e. the current belonging to $Q_{\text {int }}$ ) is only conserved in regions where $g$ is constant (see below and [3]). Hence, the KugoOjima procedure involves the (partial) adiabatic limit for $t \rightarrow-\infty$, which is difficult to control. The argument does certainly not work in nonabelian gauge theories (as can be seen by an explicit calculation of the first order of $Q_{\text {int }}$ ) [3]. We therefore prefer not to work in the adiabatic limit. The price to pay is that $Q_{\text {int }}$ does not agree with $Q$, so for the construction of the physical Hilbert space we have to check the assumptions of section 2. We do this for QED. We see no principle obstacle for the generalization to nonabelian gauge theories. But the details still need to be worked out [3].

\subsection{Free QED}

The field algebra $\mathcal{F}$ is generated by the free photon fields $A^{\mu}$ in Feynman gauge, the free spinor fields $\psi$ and $\bar{\psi}$, a pair of free ghost fields $u$ and $\tilde{u}$, the Wick monomials $j^{\mu}=: \bar{\psi} \gamma^{\mu} \psi:, \gamma_{\mu} A^{\mu} \psi, \bar{\psi} \gamma_{\mu} A^{\mu}, j_{\mu} A^{\mu}$ and the derivated free fields $\partial_{\mu} A^{\mu}$, $F^{\mu \nu}=\partial^{\mu} A^{\nu}-\partial^{\nu} A^{\mu}$. This algebra is faithfully represented on a Krein space which is given by the usual Fock space of free fields and a Krein operator which defines the indefinite inner product. The graded derivation $s$ is determined by the BRST-transformation of free fields

$$
s\left(A^{\mu}\right)=i \partial^{\mu} u, \quad s(\psi)=0, \quad s(\bar{\psi})=0, \quad s(u)=0, \quad s(\tilde{u})=-i \partial_{\mu} A^{\mu}
$$

and by translation invariance of $s$. This transformation is implemented by the free Kugo-Ojima charge [10]

$$
Q \stackrel{\text { def }}{=} \int_{x_{0}=\text { const. }} d^{3} x\left(\partial_{\nu} A^{\nu}(x)\right) \stackrel{\leftrightarrow}{\partial}_{0} u(x),
$$

which fulfills $Q^{*}=Q$, and $Q^{2}=0$. In addition the inner product $<., .>$ is positive semidefinite on $\operatorname{Ke} Q$ and the space of nullvectors in $\operatorname{Ke} Q$ is precisely $\operatorname{Ra} Q([9,13])$

\footnotetext{
${ }^{1}$ We restrict all operators (resp. formal power series of operators) to the dense invariant domain $\mathcal{D}$ and, therefore, there is no difference between symmetric and self-adjoint operators.
} 


\subsection{Construction of the interacting Kugo-Ojima charge in QED}

In QED the interaction is given by

$$
\mathcal{L}(x)=g(x): \bar{\psi}(x) \gamma_{\mu} A^{\mu}(x) \psi(x):, \quad g \in \mathcal{D}\left(\mathbf{R}^{4}\right) .
$$

We fix the double cone $\mathcal{O}$ to be the causal completion of the surface $\{(0, \mathbf{x}),|\mathbf{x}|<$ $r\}$ and assume the switching function $g \in \mathcal{D}\left(\mathbf{R}^{4}\right)$ to be constant on a neighbourhood $\mathcal{U}$ of $\overline{\mathcal{O}}(1.1)$. We study the algebra $\tilde{\mathcal{F}}(\mathcal{O})(1.5)$ of interacting fields localized in $\mathcal{O}$. The ghost fields do not couple in QED, hence $u_{\text {int }} \mathcal{L}(x)=u(x)$ and $\tilde{u}_{\text {int }} \mathcal{L}(x)=\tilde{u}(x)$ The interacting fields can be normalized such that they fulfil the field equations [8, 11]

$$
\begin{gathered}
\square A_{\text {int } \mathcal{L}}^{\mu}(x)=-g(x) j_{\text {int } \mathcal{L}}^{\mu}(x), \\
\left(i \gamma_{\mu} \partial^{\mu}-m\right) \psi_{\text {int } \mathcal{L}}(x)=-g(x)\left(\gamma_{\mu} A^{\mu} \psi\right)_{\text {int } \mathcal{L}}(x),
\end{gathered}
$$

electric current conservation

$$
\partial_{\mu} j_{\text {int } \mathcal{L}}^{\mu}=0
$$

and the following commutation relations at points $x, y \in \mathcal{O}[\mathbb{8}]$

$$
\begin{gathered}
{\left[\partial_{\mu} A_{\text {int } \mathcal{L}}^{\mu}(x), A_{\text {int } \mathcal{L}}^{\nu}(y)\right]=i \partial^{\nu} D(x-y), \quad\left[\partial_{\mu} A_{\text {int } \mathcal{L}}^{\mu}(x), \partial_{\nu} A_{\text {int } \mathcal{L}}^{\nu}(y)\right]=0} \\
{\left[\partial_{\mu} A_{\text {int } \mathcal{L}}^{\mu}(x), \psi_{\operatorname{int} \mathcal{L}}(y)\right]=D(x-y) e \psi_{\text {int } \mathcal{L}}(y)}
\end{gathered}
$$

where $D$ is the massless Pauli-Jordan distribution.

The abelian BRST-transformation $\tilde{s}=s_{0}+g s_{1}$ [1] is a graded $*$-derivation with zero square which induces the following transformations on the basic fields,

$$
\begin{aligned}
& \tilde{s}\left(A_{\text {int } \mathcal{L}}^{\mu}(x)\right)=i \partial^{\mu} u(x), \quad \tilde{s}(u(x))=0, \quad \tilde{s}(\tilde{u}(x))=-i \partial_{\mu} A_{\operatorname{int} \mathcal{L}}^{\mu}(x), \\
& \tilde{s}\left(\psi_{\operatorname{int} \mathcal{L}}(x)\right)=-e \psi_{\operatorname{int} \mathcal{L}}(x) u(x), \quad \tilde{s}\left(\bar{\psi}_{\operatorname{int} \mathcal{L}}(x)\right)=e \bar{\psi}_{\operatorname{int} \mathcal{L}}(x) u(x)
\end{aligned}
$$

for $x \in \mathcal{O}$. (The pointwise products above are well defined.)

On $\tilde{\mathcal{F}}(\mathcal{O}) \tilde{s}$ is implemented by the operator

$$
Q_{\text {int }}(g, k)=\int d^{4} x k(x)\left(\partial_{\nu} A_{\text {int } \mathcal{L}}^{\nu}(x)\right) \stackrel{\leftrightarrow}{\partial}_{0}^{x} u(x)
$$

(where $k \in \mathcal{D}(\mathcal{U})$ is a suitably chosen smeared characteristic function of the surface $\{(0, \mathbf{x}),|\mathbf{x}| \leq r\})$. Note that $\left[Q_{\text {int }}(g), F\right]_{\mp}, F \in \tilde{\mathcal{F}}(\mathcal{O})$ is independent of $k$, since the BRST-current $\partial_{\mu} A_{\text {int } \mathcal{L}}^{\mu}(x) \partial^{\leftrightarrow x}{ }_{\nu} u(x)$ is conserved within $\mathcal{U} . Q_{\text {int }}(g, k)$ is hermitian for real valued $k$ and nilpotent,

$$
\begin{gathered}
Q_{\mathrm{int}}(g, k)^{2}=\frac{1}{2}\left\{Q_{\mathrm{int}}(g, k), Q_{\mathrm{int}}(g, k)\right\}= \\
=\frac{1}{2} \int d^{4} x h(x) \int d^{4} y h(y)\left[\partial_{\mu} A_{\mathrm{int} \mathcal{L}}^{\mu}(x), \partial_{\nu} A_{\mathrm{int} \mathcal{L}}^{\nu}(y)\right] \stackrel{\leftrightarrow}{\partial}_{0}^{x} \stackrel{\leftrightarrow}{\partial}_{0} u(x) u(y)=0,
\end{gathered}
$$


by means of (3.7).

But we need in addition that the zeroth order term $Q_{0}(k)$ of $Q_{\text {int }}(g, k)(3.10)$ satisfies the positivity assumption (2.4). There seems to be no reason why this should hold for a generic choice of $k$. One might try to control the limit when $k$ tends to a smeared characteristic function of the $t=0$ hyperplane (in order that $Q_{0}(k)$ becomes equal to the free charge $\left.\mathrm{Q}(3.2)\right)$, but without an a priori information on the existence of an $\tilde{s}$-invariant state this appears to be a hard problem.

There is a more elegant way to get rid of these problems which relies on the local character of our construction. We may embed our double cone $\mathcal{O}$ isometrically into the cylinder $\mathbf{R} \times C_{L}$, where $C_{L}$ is a cube of length $L, \quad L \gg r$, with suitable boundary conditions (see sect. 4), and where the first factor denotes the time axis. If we choose the compactification length $L$ big enough, the properties of the local algebra $\tilde{\mathcal{F}}(\mathcal{O})$ are not changed.

We assume the switching function $g$ to fulfil

$$
g(x)=e=\text { constant } \quad \forall x \in \mathcal{O} \cup\left\{\left(x_{0}, \vec{x}\right)|| x_{0} \mid<\epsilon\right\} \quad(r \gg \epsilon>0)
$$

on $\mathbf{R} \times C_{L}$ and to have compact support in timelike directions. Now we may insert

$$
k(x):=h\left(x_{0}\right), \quad \text { where } \quad h \in \mathcal{D}([-\epsilon, \epsilon]), \quad \int d x_{0} h\left(x_{0}\right)=1
$$

into the expression (3.10) for $Q_{\text {int }}$. The zeroth order $Q_{0}$ then agrees with the free charge $Q$ on $C_{L}(3.2)$, hence we may apply Theorem 1 .

We emphasize that our construction shall describe QED also in the noncompactified Minkowski space (this is the main concern of the paper) and, therefore, should not depend on the compactification length $L$. On the level of the algebras this is evident. We conjecture that also the state space (i.e. the set of expectation functionals induced by vectors in the physical Hilbert space) is independent of $L$, but this remains to be proven.

An open question is the physical meaning of the remaining normalization conditions in a local perturbative construction, after the restrictions from gauge invariance and other symmetries were taken into account. The parameters involved may be considered as structure constants of the algebra of observables, but their usual interpretation as charge and mass involve the adiabatic limit.

\section{Boundary conditions for massless free gauge fields in a finite volume}

The purpose of this section is to demonstrate the importance of a suitable choice of boundary conditions. First we show that the BRST-quantization is not compatible with periodic boundary conditions for massless free gauge fields.

Let $T_{3}$ be the 3 -torus of length $L$. The algebra of a free massless scalar field $\varphi$ on $\mathbf{R} \times T_{3}$ with periodic boundary conditions is the unital *-algebra generated 
by elements $\varphi(f), f \in \mathcal{D}\left(\mathbf{R} \times T_{3}\right)$ with the relations

$$
\begin{gathered}
f \mapsto \varphi(f) \text { is linear, } \\
\varphi(\boldsymbol{\square} f)=0, \\
\varphi(f)^{*}=\varphi(\bar{f}), \\
{[\varphi(f), \varphi(g)]=\int d^{4} x d^{4} y f(x) g(y) D_{L}(x, y),}
\end{gathered}
$$

where $D_{L}$ is the fundamental solution of the wave equation on $\mathbf{R} \times T_{3}$ with periodic boundary conditions, which has the explicit form

$$
D_{L}\left(x^{0}, \vec{x}, y^{0}, \vec{y}\right)=\sum_{\vec{n} \in \mathbf{Z}^{3}} D\left(x^{0}-y^{0}, \vec{x}-\vec{y}-\vec{n}\right) .
$$

In particular one sees that $D_{L}$ coincides with $D$ (the massless commutator function on Minkowski space) on $\mathcal{O}$ if the closure of the double cone $\mathcal{O}$ is contained in $\mathbf{R} \times T_{3}$, considered as a region in Minkowski space. Hence the algebra $\mathcal{F}(\mathcal{O})$ associated to $\mathcal{O}$ is independent of the boundary conditions.

In a mode decomposition of $D_{L}$,

$$
D_{L}(x)=\frac{i}{2 L^{3}} \sum_{\vec{n} \in \mathbf{Z}^{3}, \vec{n} \neq \overrightarrow{0}} \frac{1}{\omega_{\vec{n}}}\left(e^{-i \omega_{\vec{n}} x^{0}}-e^{i \omega_{\vec{n}} x^{0}}\right) e^{i \overrightarrow{k_{\vec{n}} \vec{x}}}+\frac{x_{0}}{L^{3}},
$$

the zero mode plays a special role. The zero mode part of $\varphi(4.1-4)$ is defined by

$$
\varphi_{0}(t) \stackrel{\text { def }}{=} \frac{1}{L^{3}} \int_{T_{3}} d^{3} x \varphi(t, \vec{x}) .
$$

The algebra of the zero mode is isomorphic to the algebra of $p$ and $q$ in quantum mechanics with the free time evolution $\varphi_{0}(t)=q+p t$. There exists no ground state on this algebra.

In Feynman gauge, the components of the photon field $A_{\mu}$ are quantized as scalar fields, with a minus sign for the commutator of the zero component. The zero mode of the field $\partial_{\mu} A^{\mu}$ is then $-p^{0} L^{-3}$, which has a trivial kernel. This makes it impossible to impose the Gupta-Bleuler condition on the physical state space.

The BRST formalism is even worse. The ghost fields are quantized by

$$
(u(f)+i \tilde{u}(g))^{2}=\int d^{4} x d^{4} y f(x) g(y) D_{L}(x-y) .
$$

Inserting $f(x)=L^{-3} \delta\left(x_{0}-t_{1}\right)$ and $g(y)=-L^{-3} \delta^{\prime}\left(y_{0}-t_{2}\right)$ we obtain

$$
\left(u_{0}\left(t_{1}\right)+i \partial_{0} \tilde{u}_{0}\left(t_{2}\right)\right)^{2}=-L^{-3}
$$

\footnotetext{
${ }^{2}$ To verify (4.6) note that it is a solution of the wave equation and has the same Cauchy data as (4.5) for $x_{0}=0$.
} 
for the zero mode parts. $u_{0}$ and $\partial_{0} \tilde{u}_{0}$ are BRST invariant, hence they are observables. In addition $\left(u_{0}\left(t_{1}\right)+i \partial_{0} \tilde{u}_{0}\left(t_{2}\right)\right)$ is hermitian. We conclude that there is no nonzero (pre) Hilbert space representation of the algebra of observables.

$\left(u_{0}, \partial_{0} \tilde{u}_{0}\right)$ corresponds to a 'singlet pair' in the terminology of [14], sect. 3.1. Already there it was pointed out that the appearance of such a pair makes a consistent formulation impossible.

The way out is to choose boundary conditions which exclude the zero mode. For the electromagnetic field we may use metallic boundary conditions, i.e. the pullback of the 2 -form $F$ vanishes at the boundary (which means that the tangential components of the electric field and the normal component of the magnetic field vanish). In addition we assume that the auxiliary Nakanishi-Lautrup field $B=\partial^{\mu} A_{L \mu}$ (in Feynman gauge) satisfies Dirchlet boundary conditions. Also the ghost and antighost fields are quantized with Dirichlet boundary conditions. The details are worked out in appendix A of [8].

The BRST-quantization requires no restrictions on the boundary conditions for the electron field. For simplicity, we choose periodic boundary conditions. They have the big advantage that they are invariant under charge conjugation, hence the expectation value of the electric current (normal ordered w.r.t. the Minkowski vacuum) vanishes in the groundstate (of the torus) and, therefore, the interaction Lagrangian $\mathcal{L}(3.3)$ keeps the same form as on Minkowski space.

Acknowledgements: We profitted from discussions with Franz-Marc Boas, Izumi Ojima and Marek J. Radzikowski which are gratefully acknowledged.

\section{References}

[1] Becchi, C., Rouet, A., and Stora, R., Commun. Math. Phys. 42, 127 (1975)

Becchi, C., Rouet, A., and Stora, R., Annals of Physics (N.Y.) 98, 287 (1976)

[2] Blanchard, P., and Seneor, R., Ann. Inst. H. Poincaré A 23, 147 (1975)

[3] Boas, F.M., Dütsch, M., and K.Fredenhagen, K.," A local (perturbative) construction of observables in gauge theories: nonabelian gauge theories", work in progress

[4] Bogoliubov, N.N., and Shirkov, D.V., "Introduction to the Theory of Quantized Fields", New York (1959)

[5] Bordemann, M., and Waldmann, S., q-alg/9611004, to appear in Commun. Math. Phys.

[6] Brunetti, R., and Fredenhagen, K., "Interacting quantum fields in curved space: Renormalization of $\phi^{4}$, gr-qc/9701048, Proceedings of the Conference 'Operator Algebras and Quantum Field Theory', held at Accademia 
Nazionale dei Lincei, Rome, July 1996.

Brunetti, R., and Fredenhagen, K., "Microlocal analysis and interacting quantum field theories: Renormalization on physical backgrounds", in preparation.

[7] Brunetti, R., Fredenhagen, K., and Köhler, M., Commun. Math. Phys. 180, 312 (1996)

[8] Dütsch, M., and Fredenhagen, K., "A local (perturbative) construction of observables in gauge theories: the example of QED", preprint: hepth/9807078, DESY 98-090

[9] Dütsch, M., Hurth, T., and Scharf, G., N. Cimento A 108, 737 (1995)

[10] Dütsch, M., Hurth, T., Krahe, F., and Scharf, G., N. Cimento A 106, 1029 (1993)

[11] Dütsch, M., Krahe, F., and Scharf, G., N. Cimento A 103, 871 (1990)

[12] Epstein, H., and Glaser, V., Ann. Inst. H. Poincaré A 19, 211 (1973)

[13] Krahe, F., Acta Phys. Polonica B 27, 2453 (1996)

[14] Kugo, T., and Ojima, I., Suppl. Progr. Theor. Phys. 66, 1 (1979)

[15] Scharf, G., "Finite Quantum Electrodynamics. The causal approach", 2nd. ed., Springer-Verlag (1995)

[16] Stora, R., "Lagrangian field theory", summer school of theoretical physics about "particle physics", Les Houches, 1-79 (1971)

[17] Stora, R., "Differential algebras in Lagrangean field theory", ETH-Zürich Lectures, January-February 1993;

Popineau, G., and Stora, R., "A pedagogical remark on the main theorem of perturbative renormalization theory", unpublished preprint (1982) 